\title{
夹心型多酸修饰 $\mathrm{TiO}_{2}$ 在染料敏化太阳能电池中的应用
}

\author{
张鹤，李建平，陈维林"，王恩波* \\ 东北师范大学多酸科学教育部重点实验室, 长春 130024 \\ * 联系人, E-mail: chenwl@ nenu.edu.cn; wangeb889@ nenu.edu.cn \\ 2018-06-26 收稿, 2018-07-30 修回, 2018-08-01 接受, 2018-08-17 网络版发表 \\ 中央高校基础研究基金(2412017FZ012, 2412018QD003)和吉林省自然科学基金(20180101298JC)资助
}

\begin{abstract}
摘要染料敏化太阳能电池(DSSCs)作为一种新型的光学器件近年来受到广泛关注. 然而, 光生电子-空穴复合 严重影响染料敏化太阳能电池的光电转化效率. 多金属氧酸盐(POMs)具有优良的光电化学性质, 能够作为极好的 电子萃取剂应用于染料敏化太阳能电池光阳极中. 本文通过溶胶凝胶法将具有分子内电子转移特性的夹心型多酸 化合物 $\mathrm{K}_{15}\left\{\mathrm{~K}_{3}\left[\left(\mathrm{~A}-\alpha-\mathrm{PW}_{9} \mathrm{O}_{34}\right)_{2} \mathrm{Fe}_{2}\left(\mathrm{C}_{2} \mathrm{O}_{4}\right)_{2}\right]\right\} \cdot 29 \mathrm{H}_{2} \mathrm{O}$ 与 $\mathrm{TiO}_{2}$ 复合, 制备 $\mathrm{POM} @ \mathrm{TiO}_{2}$ 复合光阳极. 光学和电化学测试表 明, 其最低未占分子轨道 (LUMO) 能级 $(-0.06 \mathrm{~V})$ 低于 $\mathrm{TiO}_{2}$ 导带, 光学带隙为 $2.82 \mathrm{eV}$. 光伏性能测试表明基于 $\mathrm{POM} @ \mathrm{TiO}_{2} / \mathrm{P} 25$ 复合光阳极的 $\mathrm{DSSC}$ 效率达到了 $6.33 \%$, 相比于纯 $\mathrm{TiO}_{2}$ 光阳极电池 $(5.52 \%)$ 提高了 $15 \%$. 电化学阻抗 谱(EIS)、暗电流测试和开路电压衰减测试证明夹心型多酸化合物有效地抑制电子-空穴复合，增长电子寿命。入射 单色光子-电子转化效率(IPCE)测试进一步证明此多酸的引入增强了单色光转化效率(从 $35 \%$ 提高到 $53 \%$ ).
\end{abstract}

关键词染料敏化太阳能电池, 多金属氧酸盐, 光阳极, 电子传输, 电子-空穴复合

随着人们对生活的高质量要求，对能源的需求 量越来越大. 现今, 化石能源的急剧消耗导致了全球 能源紧缺. 同时, 化石燃料的燃烧也会带来温室效 应，全球空气质量的下降，酸雨的形成及水体污染等 一系列问题. 由于人们对自然的开发程度加大, 不可 再生能源被大量消耗, 因此是否能够寻找到可以替 代化石能源的新能源成为了人们日益关注的问 题 ${ }^{[1 \sim 4]}$. 可再生能源中, 太阳能是我们普遍使用的清 洁能源之一. 太阳能电池主要有硅太阳能电池、多晶 体薄膜太阳能电池、染料敏化太阳能电池、有机聚合 物太阳能电池等. 其中染料敏化太阳能电池(DSSCs) 具有低价、制作简单以及高效率的优势 ${ }^{[1]}$. 其组成为 光阳极、氧化还原电解质和对电极. 由于光阳极可以 吸附染料并能影响电子的传输, 因此在DSSCs中起 着至关重要的作用. 光阳极上的半导体材料主要由 一些具有纳米尺寸或纳米结构的二元及多元化合物
组成. 所研究的DSSCs中, 以宽带的氧化物半导体作 为光阳极的数量较多. 光阳极的种类、价带结构、表 面形貌等因素都会对DSSCs的效率产生很大的影响。 目前可用作光阳极的半导体材料主要有 $\mathrm{TiO}_{2}, \mathrm{ZnO}$, $\mathrm{SnO}_{2}, \mathrm{Nb}_{2} \mathrm{O}_{5}, \mathrm{In}_{2} \mathrm{O}_{3}, \mathrm{Zn}_{2} \mathrm{SnO}_{4}, \mathrm{SrTiO}_{3}, \mathrm{Fe}_{2} \mathrm{O}_{3}, \mathrm{CdS}$, $\mathrm{WO}_{3}, \mathrm{Ta}_{2} \mathrm{O}_{3}{ }^{[5 \sim 11]}$. 其中, 宽带隙半导体 $\mathrm{TiO}_{2}$ 具有成本 低、无毒且化学性质稳定的优势.2014年Mathew等 人 $^{[12]}$ 在Nature上报道以 $\mathrm{TiO}_{2}$ 做光阳极制作的染料敏 化电池，效率达到了 $13 \%$. 然而，当DSSCs中的敏化 剂被光激发后, 产生的光生电子立即注人到半导体 的导带. 在这个过程中, 电子通常可以与染料和电解 质重新结合，使电池的效率降低. 因此针对于这个问 题，研究者进行了对光阳极形态的控制，金属改性与 其他材料进行复合等研究 ${ }^{[13,14]}$, 以改善光阳极的电 子传输效率.

多金属氧酸盐 $(\mathrm{POMs})$ 是一类具有铇、钿或钒的 
最高氧化态的金属氧簇, 由于其结构的多样性和独 特的物理化学性质, 在催化、光化学、生物学和医学 领域具有广泛的应用前景 ${ }^{[15]}$. POMs 作为电子传输体 已被广泛应用在DSSCs中. 在DSSCs中, 染料N719光 致激发, 产生的光生电子注人到半导体 $\mathrm{TiO}_{2}$ 中, 电子 经 $\mathrm{TiO}_{2}$ 再传递给多酸, 最后进人外电路. POMs 可传 递电子的这种特性可以很大程度地提高电池的光电 转化效率. 目前有多种多酸被运用在太阳能电池的 光学器件中, 并取得了很好的效果. 2007年Yoon课题 组 ${ }^{[16]}$ 用旋涂的方式将含铇的多金属盐酸盐均匀地旋 涂在 $\mathrm{TiO}_{2}$ 阳极膜上. 该含铇的多金属氧酸盐作为一 种良好的电子受体, 可有效地将敏化剂产生的光生 电子传递到外电路, 并能抑制电子-空穴的复合, 使 电池效率提高了 $23 \% .2009$ 年, $\mathrm{Xu}$ 等人 ${ }^{[17]}$ 报道了将多 酸 $\mathrm{SiMo}_{12}$ 和有机物质酞菁用层接层 $(\mathrm{LbL})$ 的方法制备 光阳极, 复合膜相较于单独使用的铜酞菁, 光电转换 效率明显增强. 这主要是因为多酸 $\mathrm{SiMo}_{12}$ 的掺人使 酞菁被激发出的电子可以更好地传递到外电路, 促 进了电子和空穴的有效分离. 2013年本课题组 ${ }^{[18]}$ 首 次将开普勒型多酸 $\left\{\mathrm{W}_{72} \mathrm{~V}_{30}\right\}$ 应用在DSSCs中, 产生了 一定的光电响应; 2017 年本课题组 ${ }^{[19]}$ 将夹心型多酸 $\mathrm{K}_{6} \mathrm{Na}_{2} \mathrm{Ni}_{2}\left[\mathrm{Ni}_{4}\left(\mathrm{H}_{2} \mathrm{O}\right)_{2}\left(\mathrm{SiW}_{9} \mathrm{O}_{34}\right)_{2}\right] \cdot 30 \mathrm{H}_{2} \mathrm{O}$ 引人光阳极, 使电流密度及电池效率有效提高, 证明了供体-受体 (donor-acceptor) 型多酸以及开普勒型多酸可以有效 地抑制电子和空穴复合, 并延长电子寿命.

夹心型POMs 是目前被广泛研究的功能性 POM, 其磁性、催化性质以及光电性能已被深人研究. 我们 通过循环伏安和固体紫外漫反射计算出该夹心型多 酸化合物 $\mathrm{K}_{15}\left\{\mathrm{~K}_{3}\left[\left(\mathrm{~A}-\alpha-\mathrm{PW}_{9} \mathrm{O}_{34}\right)_{2} \mathrm{Fe}_{2}\left(\mathrm{C}_{2} \mathrm{O}_{4}\right)_{2}\right]\right\} \cdot 29 \mathrm{H}_{2} \mathrm{O}$ 的最高占有轨道 (HOMO) 和最低未占分子轨道 (LUMO) 能级, 发现该多酸化合物的LUMO能级低于 $\mathrm{TiO}_{2}$. 因此光照时, N719产生的光生电子可以传递到 $\mathrm{TiO}_{2}$ 上, 再经多酸化合物传递到外电路, 在整个过程 中多酸化合物能起到电子传输体的作用. 本文通过 溶胶凝胶法将夹心型 $\mathrm{POM} \mathrm{K}_{15}\left\{\mathrm{~K}_{3}\left[\left(\mathrm{~A}-\alpha-\mathrm{PW}_{9} \mathrm{O}_{34}\right)_{2} \mathrm{Fe}_{2}-\right.\right.$ $\left.\left.\left(\mathrm{C}_{2} \mathrm{O}_{4}\right)_{2}\right]\right\} \cdot 29 \mathrm{H}_{2} \mathrm{O}$ 引入DSSCs的光阳极中, 对修饰前后 的阳极膜组装的电池测试了电流密度-电压 $(J-V)$ 曲 线, 并计算了它们的效率. 结果表明, P25膜制作的 DSSCs 的效率是 $5.52 \%$, 而用POM@ $\mathrm{TiO}_{2} / \mathrm{P} 25$ 光阳极 制作的DSSC效率达到了 $6.33 \%$, 与前者相比提高了 $15 \%$. POM@ $\mathrm{TiO}_{2} / \mathrm{P} 25$ 组装成电池, 效率比P25阳极膜 明显增高. 此外, 还通过测试DSSCs的阻抗, 开路电
压衰退曲线以及暗电流曲线, 证明了这种多酸化合物 具有抑制电子-空穴复合以及延长电子寿命的作用.

\section{1 材料与方法}

(i) 原料与仪器. $\mathrm{K}_{3}\left[\mathrm{Fe}\left(\mathrm{C}_{2} \mathrm{O}_{4}\right)_{3}\right] \cdot 3 \mathrm{H}_{2} \mathrm{O}, \mathrm{Na}_{2} \mathrm{WO}_{4}$. $2 \mathrm{H}_{2} \mathrm{O}$, 磷酸、冰醋酸、P25(德国)、乙醇、松油醇、乙 基纤维素、硫酸钠、硫酸钡、氧化铝抛光粉、氯化钾; Alpha Centaurt FTIR红外光谱仪、紫外-可见分光光度 计(SHIMADZU UV-2600)、Rigaku D/MAX-2550粉末 衍射仪 (XRD)、SU8010扫描电子显微镜 (SEM)、 PerkinElmer TGA7热分析仪、太阳能电池光谱响应测 试系统(IPCE)、电化学工作站(上海辰华CHI601D)、恒 电位仪.

(ii ) $\mathrm{Na}_{9}\left[\mathrm{~A}-\mathrm{PW}_{9} \mathrm{O}_{34}\right] \cdot 7 \mathrm{H}_{2} \mathrm{O}$ 和 $\mathrm{K}_{15}\left\{\mathrm{~K}_{3}\left[\left(\mathrm{~A}-\alpha-\mathrm{PW}_{9} \mathrm{O}_{34}\right)_{2}\right.\right.$ $\mathrm{Fe}_{2}\left(\mathrm{C}_{2} \mathrm{O}_{4}\right)_{2}$ ] $\} \cdot 29 \mathrm{H}_{2} \mathrm{O}$ 的合成. 根据文献 [20] 合成 $\mathrm{Na}_{9}\left[\mathrm{~A}-\mathrm{PW}_{9} \mathrm{O}_{34}\right] \cdot 7 \mathrm{H}_{2} \mathrm{O}$ 和 $\mathrm{K}_{15}\left\{\mathrm{~K}_{3}\left[\left(\mathrm{~A}-\alpha-\mathrm{PW}_{9} \mathrm{O}_{34}\right)_{2} \mathrm{Fe}_{2}\right.\right.$ $\left.\left.\left(\mathrm{C}_{2} \mathrm{O}_{4}\right)_{2}\right]\right\} \cdot 29 \mathrm{H}_{2} \mathrm{O}$. 详细过程如下: 将 $30 \mathrm{~g} \mathrm{Na}_{2} \mathrm{WO}_{4} \cdot 2 \mathrm{H}_{2} \mathrm{O}$ 溶解于 $37.5 \mathrm{~mL}$ 蒸馏水中, 不断摚拌, 直至固体完全 溶解, 然后逐滴加人 $1 \mathrm{~mol} \mathrm{~L}^{-1} 85 \%$ 的磷酸, 将溶液的 $\mathrm{pH}$ 调节到 8 9, 剧烈搅拌, 同时将 $5.6 \mathrm{~mL}$ 的冰醋酸逐 滴加人到溶液中, 产生大量白色沉淀, 持续搅拌 $1 \mathrm{~h}$, 抽滤、室温下干燥, 产量为 $19.1 \mathrm{~g}$.

取 $0.356 \mathrm{~g}$ 的 $\mathrm{K}_{3}\left[\mathrm{Fe}\left(\mathrm{C}_{2} \mathrm{O}_{4}\right)_{3}\right] \cdot 3 \mathrm{H}_{2} \mathrm{O}$ 溶解在 $6 \mathrm{~mL}$ 蒸馏 水中, 并加热至 $60^{\circ} \mathrm{C}$, 搅拌, 得到翠绿色溶液. 称取 $\mathrm{Na}_{9}\left[\mathrm{~A}-\mathrm{PW}_{9} \mathrm{O}_{34}\right] \cdot 7 \mathrm{H}_{2} \mathrm{O} \quad 0.774 \mathrm{~g}$, 加人上述翠绿色溶液 中, 不断搅拌, 溶液的颜色由翠绿色变为橄榄绿, 将 溶液趁热抽滤, 得到的滤液放在敞口容器中, 置于暗 处 $2 \mathrm{~h}$ 后, 溶液中出现亮黄绿色的针状晶体, 抽滤, 并 用蒸馏水洗涤 ${ }^{[20]}$.

(iii) 光阳极的制备. 将 $5 \mathrm{~mL}$ 钛酸异丙酯和 $3 \mathrm{~mL}$ 正丁醇混合, 在 $45^{\circ} \mathrm{C}$ 下搅拌加热 $3 \mathrm{~h}$, 随后称取 $0.05 \mathrm{~g} \mathrm{~K}_{15}\left\{\mathrm{~K}_{3}\left[\left(\mathrm{~A}-\alpha-\mathrm{PW}_{9} \mathrm{O}_{34}\right)_{2} \mathrm{Fe}_{2}\left(\mathrm{C}_{2} \mathrm{O}_{4}\right)_{2}\right]\right\} \cdot 29 \mathrm{H}_{2} \mathrm{O}$ 多酸 将其溶于 $8 \mathrm{~mL}$ 水中, 将多酸的水溶液缓慢加人钛酸 异丙酯和正丁醇的混合溶液中, 在 $80^{\circ} \mathrm{C}$ 加热 $3 \mathrm{~h}$. 将 制得的混合物 $80^{\circ} \mathrm{C}$ 干燥 $12 \mathrm{~h}$. 将干燥完毕的混合物水 洗3次, $80^{\circ} \mathrm{C}$ 干燥, 得到POM@ $\mathrm{TiO}_{2}$ 复合物.

根据文献[22,23]制备 $\mathrm{TiO}_{2}$ 浆料: 称取 $0.57 \mathrm{~g} \mathrm{TiO}_{2}$ 粉末及 $0.03 \mathrm{~g} \mathrm{POM@} \mathrm{TiO}_{2}$ 复合物加人研钵, 并用移液 枪量取 $100 \mu \mathrm{L}$ 的乙酸, 研磨 $5 \mathrm{~min}$, 然后加人蒸馏水 $100 \mu \mathrm{L}$ 研磨 $1 \mathrm{~min}$, 重复5次, 加人 $100 \mu \mathrm{L}$ 乙醇, 研磨 $1 \mathrm{~min}$, 共重复 15 次; 再分 6 次加人 $250 \mu \mathrm{L}$ 的乙醇, 每 次研磨 $1 \mathrm{~min}$. 用适量乙醇将 $\mathrm{TiO}_{2}$ 浆料转移到烧杯中, 
搅拌 $1 \mathrm{~min}$, 超声 $1 \mathrm{~min}$, 再摚拌 $1 \mathrm{~min}$; 然后, 在搅拌 的同时, 加人 $2.24 \mathrm{~mL}$ 松油醇, 搅拌 $1 \mathrm{~min}$, 超声 $1 \mathrm{~min}$, 再摚拌 $1 \mathrm{~min}$, 加人 $20 \mathrm{~mL}$ 乙基纤维素的乙醇溶液, 摚 拌 $1 \mathrm{~min}$, 超声 $1 \mathrm{~min}, 80^{\circ} \mathrm{C}$ 加热浆料, 使乙醇蒸干.

导电玻璃(FTO)的清洗: 加人洗涤剂超声 $30 \mathrm{~min}$, 然后用水将洗涤剂的残留泡沫冲洗干净, 加人异丙醇 超声 $30 \mathrm{~min}$, 再加人乙醇超声 $30 \mathrm{~min}$; 将超声完毕的 FTO单片取出, $\mathrm{N}_{2}$ 吹干, 用丝网印刷法将上步制得的 浆料刮在FTO上, 然后浸泡染料N719, 制做光阳极.

(iv) 组装电池。以N719染料敏化的POM@ $\mathrm{TiO}_{2} / \mathrm{P} 25$ 纳米多孔薄膜为光阳极, 以铂片为对电极, 将染料敏化的光阳极以及对电极用夹子固定, 并在 其间隙中滴人电解质 $\left(0.1 \mathrm{~mol} \mathrm{~L}^{-1} \mathrm{LiI}, 0.05 \mathrm{~mol} \mathrm{~L}^{-1} \mathrm{I}_{2}\right.$, $0.6 \mathrm{~mol} \mathrm{~L}^{-1} 1,2$-二甲基-3-丙基咪唑碘和 $0.5 \mathrm{~mol} \mathrm{~L}^{-1} 4$ 叔丁基丙腈)得到染料敏化太阳能电池.

( V) 电池的光电性能测试. 采用500 W氙灯为 太阳光模拟光源, 其人射光强 $P_{\mathrm{in}}$ 为 $100 \mathrm{~mW} \mathrm{~cm}{ }^{-2}$; 室 温下, 采用电化学工作站进行测试, 测试DSSCs的暗 电流曲线、循环伏安曲线、开路电压衰退曲线及 $J-V$ 曲线; 记录 $\mathrm{DSSC}$ 的电流密度 $J_{\mathrm{sc}}$ 以及开路电压 $V_{\mathrm{oc}}$, 并 计算其填充因子 $F F$ 以及光电转换效率 $\eta$; 恒电位仪测 量DSSCs的交流阻抗谱(EIS); 太阳能电池光谱响应 测试系统测试DSSCs的人射单色光子-电子转换效率 (IPCE).

(vi) 表征与分析. 采用Alpha Centaurt FTIR红外 光谱仪测量夹心型多酸化合物的红外光谱, 用 $\mathrm{KBr}$ 压 片法, 波长范围为 400 4000 $\mathrm{cm}^{-1}$; PerkinElmer TGA7 热分析仪测试多酸的热重(TG)和差热(DTA)曲线; 用 紫外-可见分光光度计SHIMADZU UV-2600测试夹心
型多酸化合物的固体紫外漫反射曲线, 硫酸钡作为 标准参比，测试的波长范围为200 800 $\mathrm{nm}$; 使用 Rigaku D/MAX-3 粉末衍射仪测试夹心型多酸化合 物的XRD, 测试条件为 $\mathrm{Cu} / \mathrm{Pd} \mathrm{K \alpha}(\lambda=1.5418 \AA)$, 室温, $2 \theta=5^{\circ} \sim 80^{\circ}$; 用 SU8010扫描电子显微镜观察复合膜 的表面形貌及元素分布.

\section{2 结果与讨论}

\section{1 夹心型多酸的表征}

图 1(a) 为夹心型多酸 $\mathrm{K}_{15}\left\{\mathrm{~K}_{3}\left[\left(\mathrm{~A}-\alpha-\mathrm{PW}_{9} \mathrm{O}_{34}\right)_{2} \mathrm{Fe}_{2}\right.\right.$ $\left.\left.\left(\mathrm{C}_{2} \mathrm{O}_{4}\right)_{2}\right]\right\} \cdot 29 \mathrm{H}_{2} \mathrm{O}$ 的XRD谱，可以看出本文合成的这 种多酸与文献[20]报道的XRD衍射峰基本一致. 图 1(b)为本文合成的 $P O M s$ 的红外图, $\mathrm{Na}_{9}\left[\mathrm{~A}-\mathrm{PW}_{9} \mathrm{O}_{34}\right] \cdot 7 \mathrm{H}_{2} \mathrm{O}$ 特征吸收峰 1052(s) $\mathrm{cm}^{-1}$ 归属于 $\mathrm{W}-\mathrm{O}_{\mathrm{a}}$ 键的吸收峰, 941(s) $\mathrm{cm}^{-1}$ 归属于 $\mathrm{W}=\mathrm{O}_{\mathrm{d}}$ 键的吸收峰, $886(\mathrm{~m}), 836(\mathrm{~s})$ $\mathrm{cm}^{-1}$ 归属于 $\mathrm{W}-\mathrm{O}_{\mathrm{b}}-\mathrm{W}$ 的特征吸收峰, $760(\mathrm{~s}) \mathrm{cm}^{-1}$ 归属 于 $\mathrm{W}-\mathrm{O}_{\mathrm{c}}-\mathrm{W}$ 的吸收峰; $\mathrm{K}_{15}\left\{\mathrm{~K}_{3}\left[\left(\mathrm{~A}-\alpha-\mathrm{PW}_{9} \mathrm{O}_{34}\right)_{2} \mathrm{Fe}_{2}\right.\right.$ $\left.\left.\left(\mathrm{C}_{2} \mathrm{O}_{4}\right)_{2}\right]\right\} \cdot 29 \mathrm{H}_{2} \mathrm{O}$ 特征吸收峰 $1665.1(\mathrm{~m}) \mathrm{cm}^{-1}$ 属于 $\mathrm{C}=\mathrm{O}$ 的伸缩振动吸收, $1281.4(\mathrm{~s}) \mathrm{cm}^{-1}$ 属于 $\mathrm{C}-\mathrm{O}$ 伸缩以及 $-\mathrm{O}-\mathrm{C}=\mathrm{O}$ 弯曲震动, $1067.6(\mathrm{~m}) \mathrm{cm}^{-1}$ 归属于 $\mathrm{W}-\mathrm{O}_{\mathrm{a}}$ 键的 吸收峰, 930. $1(\mathrm{~m}) \mathrm{cm}^{-1}$ 归属于 $\mathrm{W}=\mathrm{O}_{\mathrm{d}}$ 键的吸收峰, 883.1(w), $843.2(\mathrm{w}) \mathrm{cm}^{-1}$ 归属于 $\mathrm{W}-\mathrm{O}_{\mathrm{b}}-\mathrm{W}$ 的特征吸 收峰。

图 1(c)为夹心型多酸 $\mathrm{K}_{15}\left\{\mathrm{~K}_{3}\left[\left(\mathrm{~A}-\alpha-\mathrm{PW}_{9} \mathrm{O}_{34}\right)_{2} \mathrm{Fe}_{2}\right.\right.$ $\left.\left.\left(\mathrm{C}_{2} \mathrm{O}_{4}\right)_{2}\right]\right\} \cdot 29 \mathrm{H}_{2} \mathrm{O}$ 在空气气氛中轱烧的 TG和DTA曲线. 很明显, 夹心型 $\mathrm{POM} \mathrm{K} \mathrm{K}_{15}\left\{\mathrm{~K}_{3}\left[\left(\mathrm{~A}-\alpha-\mathrm{PW}_{9} \mathrm{O}_{34}\right)_{2} \mathrm{Fe}_{2}\right.\right.$ $\left.\left.\left(\mathrm{C}_{2} \mathrm{O}_{4}\right)_{2}\right]\right\} \cdot 29 \mathrm{H}_{2} \mathrm{O}$ 的 TG曲线出现了2步失重: 第1步失 重在 $100^{\circ} \mathrm{C}$ 以下，第2步失重在 $100 \sim 400^{\circ} \mathrm{C}$, 在这个过
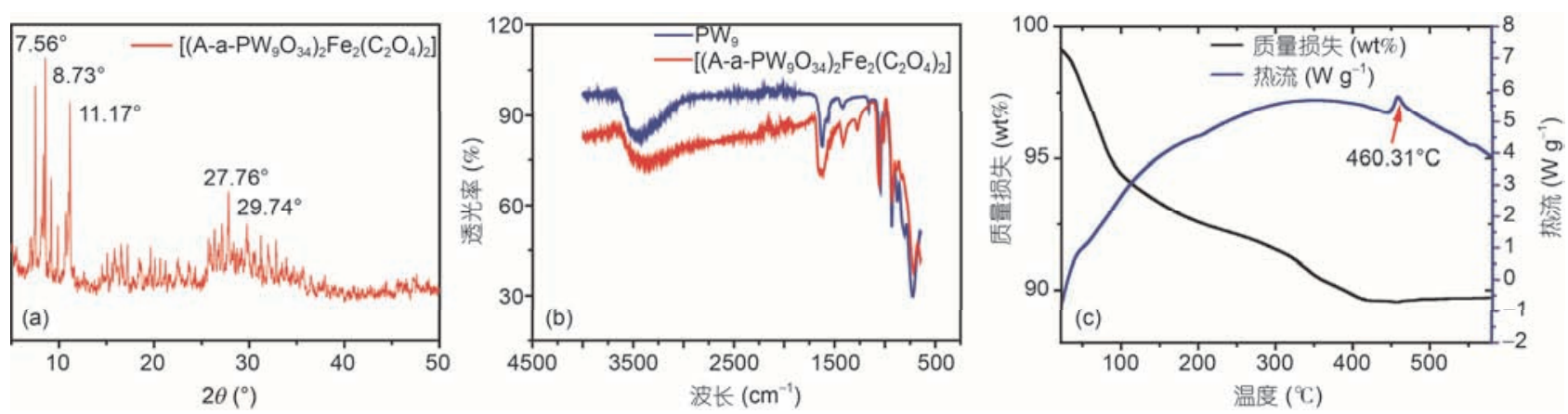

图 1 (网络版彩色)夹心型多酸的晶体表征. (a) 夹心型多酸 $\mathrm{K}_{15}\left\{\mathrm{~K}_{3}\left[\left(\mathrm{~A}-\alpha-\mathrm{PW}_{9} \mathrm{O}_{34}\right)_{2} \mathrm{Fe}_{2}\left(\mathrm{C}_{2} \mathrm{O}_{4}\right)_{2}\right]\right\} \cdot 29 \mathrm{H}_{2} \mathrm{O}$ 的XRDi谱; (b) 多酸 $\mathrm{Na}_{9}\left[\mathrm{~A}-\mathrm{PW} \mathrm{PW}_{34}\right] \cdot 7 \mathrm{H}_{2} \mathrm{O}$ 和 $\mathrm{K}_{15}\left\{\mathrm{~K}_{3}\left[\left(\mathrm{~A}-\alpha-\mathrm{PW}_{9} \mathrm{O}_{34}\right)_{2} \mathrm{Fe}_{2}\left(\mathrm{C}_{2} \mathrm{O}_{4}\right)_{2}\right]\right\} \cdot 29 \mathrm{H}_{2} \mathrm{O}$ 的红外谱图; (c) $\mathrm{K}_{15}\left\{\mathrm{~K}_{3}\left[\left(\mathrm{~A}-\alpha-\mathrm{PW}_{9} \mathrm{O}_{34}\right)_{2} \mathrm{Fe}_{2}\left(\mathrm{C}_{2} \mathrm{O}_{4}\right)_{2}\right]\right\} \cdot 29 \mathrm{H}_{2} \mathrm{O}$ 的TG以及DTA曲线

Figure 1 (Color online) Crystalline characterization of sandwich-type POM. (a) The XRD spectrum of sandwich-type POM $\mathrm{K}_{15}\left\{\mathrm{~K}_{3}\left[\left(\mathrm{~A}-\alpha-\mathrm{PW}_{9} \mathrm{O}_{34}\right)_{2}\right.\right.$ $\left.\left.\mathrm{Fe}_{2}\left(\mathrm{C}_{2} \mathrm{O}_{4}\right)_{2}\right]\right\} \cdot 29 \mathrm{H}_{2} \mathrm{O}$. (b) IR spectra of $\mathrm{Na}_{9}\left[\mathrm{~A}-\mathrm{PW}_{9} \mathrm{O}_{34}\right] \cdot 7 \mathrm{H}_{2} \mathrm{O}$ and $\mathrm{K}_{15}\left\{\mathrm{~K}_{3}\left[\left(\mathrm{~A}-\alpha-\mathrm{PW}_{9} \mathrm{O}_{34}\right)_{2} \mathrm{Fe}_{2}\left(\mathrm{C}_{2} \mathrm{O}_{4}\right)_{2}\right]\right\} \cdot 29 \mathrm{H}_{2} \mathrm{O}$. (c) TG and DTA curves of $\mathrm{K}_{15}\left\{\mathrm{~K}_{3}[(\mathrm{~A}-\alpha-\right.$ $\left.\left.\left.\mathrm{PW}_{9} \mathrm{O}_{34}\right)_{2} \mathrm{Fe}_{2}\left(\mathrm{C}_{2} \mathrm{O}_{4}\right)_{2}\right]\right\} \cdot 29 \mathrm{H}_{2} \mathrm{O}$ 
程中多酸共失重 $10 \%$, 这可能是由于多酸分子失去 了结晶水. 而在DTA曲线中, 约 $460.31{ }^{\circ} \mathrm{C}$ 处出现放热 峰. 以上结果说明多酸在 $460.31{ }^{\circ} \mathrm{C}$ 分解.

\section{2 夹心型多酸的能级测定}

通过循环伏安法测试该多酸化合物的氧化还原 性能, 采取三电极体系, 以玻碳电极为工作电极、铂 丝为对电极、 $\mathrm{Ag} / \mathrm{AgCl}$ 电极为参比电极, 电解质为 1 $\mathrm{mol} / \mathrm{L} \mathrm{Na} \mathrm{SO}_{4}$ 溶液, 测试结果如图2(a)所示. 因为多 酸及多酸衍生物的LUMO是以铇原子为中心的 $\mathrm{d}$ 轨道 的组合, 因此通过第 1 个还原电位 $(-0.25 \mathrm{~V})$ 来计算多 酸化合物的 LUMO 能级, 其计算公式为 $E_{\mathrm{NHE}}=$ $E_{\mathrm{Ag} / \mathrm{AgCl}}+0.19^{[21]}$, 经计算该多酸化合物的 $\mathrm{LUMO}$ 能级 为 $-0.06 \mathrm{~V}$.

通过固体紫外漫反射对多酸 $\mathrm{K}_{15}\left\{\mathrm{~K}_{3}\left[\left(\mathrm{~A}-\alpha-\mathrm{PW}_{9} \mathrm{O}_{34}\right)_{2}\right.\right.$ $\left.\left.\mathrm{Fe}_{2}\left(\mathrm{C}_{2} \mathrm{O}_{4}\right)_{2}\right]\right\} \cdot 29 \mathrm{H}_{2} \mathrm{O}$ 的带隙进行估算. 利用 $\left[F\left(R_{\infty}\right) h v\right]^{2}$ 对 $h v\left(h v=1240 / \lambda, R_{\infty}=R_{\infty}\right.$ (样品) $/ R_{\infty}$ (参比) ) 作图得到 Kubelka-Munk曲线. 得到函数图后, 如图2(b)所示作 切线, 切线与横坐标的交点即为禁带带隙 $(2.82 \mathrm{eV})$, 最后得到 $\mathrm{TiO}_{2}$ 和该多酸化合物的能级图2(b). 由图可 知, 多酸 $\mathrm{K}_{15}\left\{\mathrm{~K}_{3}\left[\left(\mathrm{~A}-\alpha-\mathrm{PW}_{9} \mathrm{O}_{34}\right)_{2} \mathrm{Fe}_{2}\left(\mathrm{C}_{2} \mathrm{O}_{4}\right)_{2}\right]\right\} \cdot 29 \mathrm{H}_{2} \mathrm{O}$ 的 LUMO能级为 $-0.06 \mathrm{~V}$, 低于 $\mathrm{TiO}_{2}$ 的导带, 意味着染 料的光生电子可以传递到 $\mathrm{TiO}_{2}$ 和多酸上, 从而进人 外电路, 多酸可以阻止传递到 $\mathrm{TiO}_{2}$ 的光生电子回流 发生电子-空穴复合.

\section{3 夹心型多酸化合物修饰的光阳极形貌及组成}

为了研究多酸对 $\mathrm{TiO}_{2}$ 形貌的影响, 对复合膜进 行了元素分布(Mapping)和SEM测试. X射线能量色散
谱(EDS)可知, 复合膜上具有 $\mathrm{C}, \mathrm{O}, \mathrm{P}, \mathrm{Fe}, \mathrm{Ti}, \mathrm{W}$ 元素, 元素含量如图3(a)所示. 从 Mapping分析中得出, C, $\mathrm{O}, \mathrm{P}, \mathrm{Fe}, \mathrm{Ti}, \mathrm{W}$ 元素在 $\mathrm{TiO}_{2}$ 复合膜的分布如图3(b) (g) 所示. 由图可知, 多酸 $\mathrm{K}_{15}\left\{\mathrm{~K}_{3}\left[\left(\mathrm{~A}-\alpha-\mathrm{PW}_{9} \mathrm{O}_{34}\right)_{2} \mathrm{Fe}_{2}\right.\right.$ $\left.\left.\left(\mathrm{C}_{2} \mathrm{O}_{4}\right)_{2}\right]\right\} \cdot 29 \mathrm{H}_{2} \mathrm{O}$ 在 $\mathrm{TiO}_{2}$ 膜上均匀分布, 说明夹心型 多酸复合物成功地修饰了 $\mathrm{TiO}_{2}$ 阳极膜.

图 4 是 P25 和 POM@ $\mathrm{TiO}_{2} / \mathrm{P} 25$ 的 SEM 图, 多酸修 饰前后, P25阳极膜的形貌并没有发生明显的变化, 说明多酸对二氧化钛的形貌并没有产生影响.

$\mathrm{POM} @ \mathrm{TiO}_{2} / \mathrm{P} 25$ 复合材料和锐钛矿 $\mathrm{TiO}_{2}$ 的 XRD 谱图如图 5所示. 从图中可以看出, 二者的衍射峰位 置以及强弱基本一致, 且与锐钛矿标准卡片 (JCPDS No. 21-1272)的峰位置一致, 这说明复合材料中的 $\mathrm{TiO}_{2}$ 在炦烧后成功地转变为锐钛矿晶型. 在复合材料的 $\mathrm{XRD}$ 谱中, 未观察到多酸 $\mathrm{K}_{15}\left\{\mathrm{~K}_{3}\left[\left(\mathrm{~A}-\alpha-\mathrm{PW}_{9} \mathrm{O}_{34}\right)_{2} \mathrm{Fe}_{2}\right.\right.$ $\left.\left.\left(\mathrm{C}_{2} \mathrm{O}_{4}\right)_{2}\right]\right\} \cdot 29 \mathrm{H}_{2} \mathrm{O}$ 的衍射峰, 这可能是由于多酸在复 合材料中的含量相对较低的缘故.

\subsection{DSSCs的光电性能}

在AM1.5 $100 \mathrm{~mW} / \mathrm{cm}^{2}$ 的光照条件下测试了复合 膜以及 $\mathrm{P} 25$ 光阳极制作的DSSCs光伏性能, 图6(a)展 示了二者的光电流密度-电压特性曲线, 其 $J_{\mathrm{sc}}, V_{\mathrm{oc}}$, $F F$ 和 $\eta$ 列于表 1 , 这些数据是 10 次测试的平均值. 很 明显, $\mathrm{POM} @ \mathrm{TiO}_{2} / \mathrm{P} 25$ 复合膜组装的DSSC的光电转 化性能最佳. 复合膜制作的DSSC其电池效率达到了 $6.33 \%$, 与P 25光阳极组装的DSSC效率 $(5.52 \%)$ 相比, 光电转换效率提高了 $15 \%$. 说明将夹心型多酸 $\mathrm{K}_{15}\left\{\mathrm{~K}_{3}\left[\left(\mathrm{~A}-\alpha-\mathrm{PW}_{9} \mathrm{O}_{34}\right)_{2} \mathrm{Fe}_{2}\left(\mathrm{C}_{2} \mathrm{O}_{4}\right)_{2}\right]\right\} \cdot 29 \mathrm{H}_{2} \mathrm{O}$ 引人 $\mathrm{TiO}_{2}$ 半导体, 多酸可作为电子受体接受染料激发的电子,
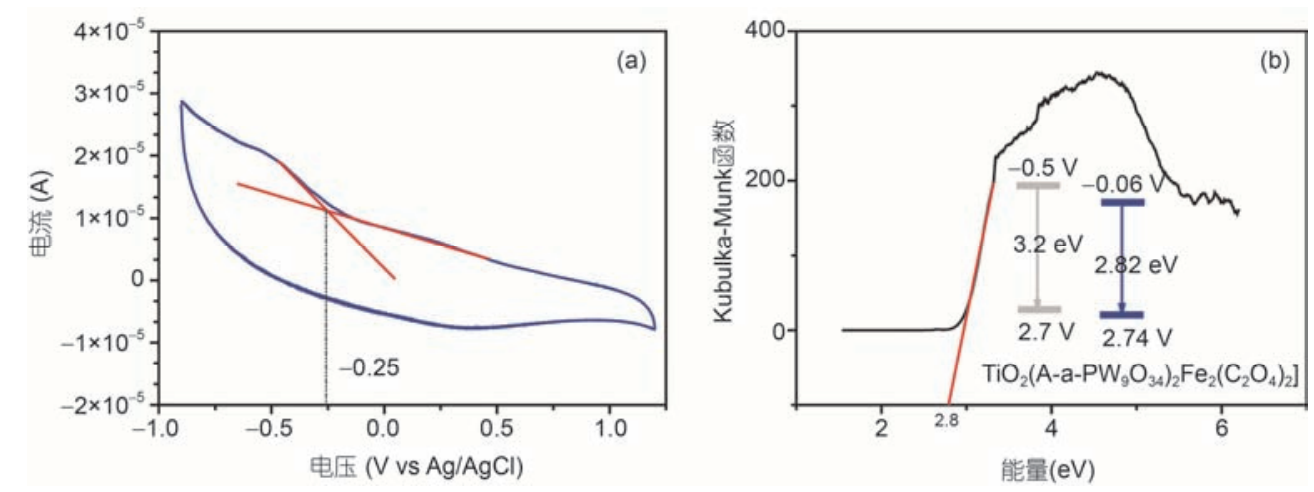

图 2 (网络版彩色)夹心型多酸的能级测定. (a) 循环伏安曲线; (b) Kubelka Munk-E曲线以及 $\mathrm{TiO}_{2}$ 及夹心型POM的能级示意图

Figure 2 (Color online) Energy level calculation of sandwich-type POM. (a) The cyclic voltammetry curve. (b) The Kubelka Munk-E curve and the energy levels of $\mathrm{TiO}_{2}$ and sandwich-type POM 

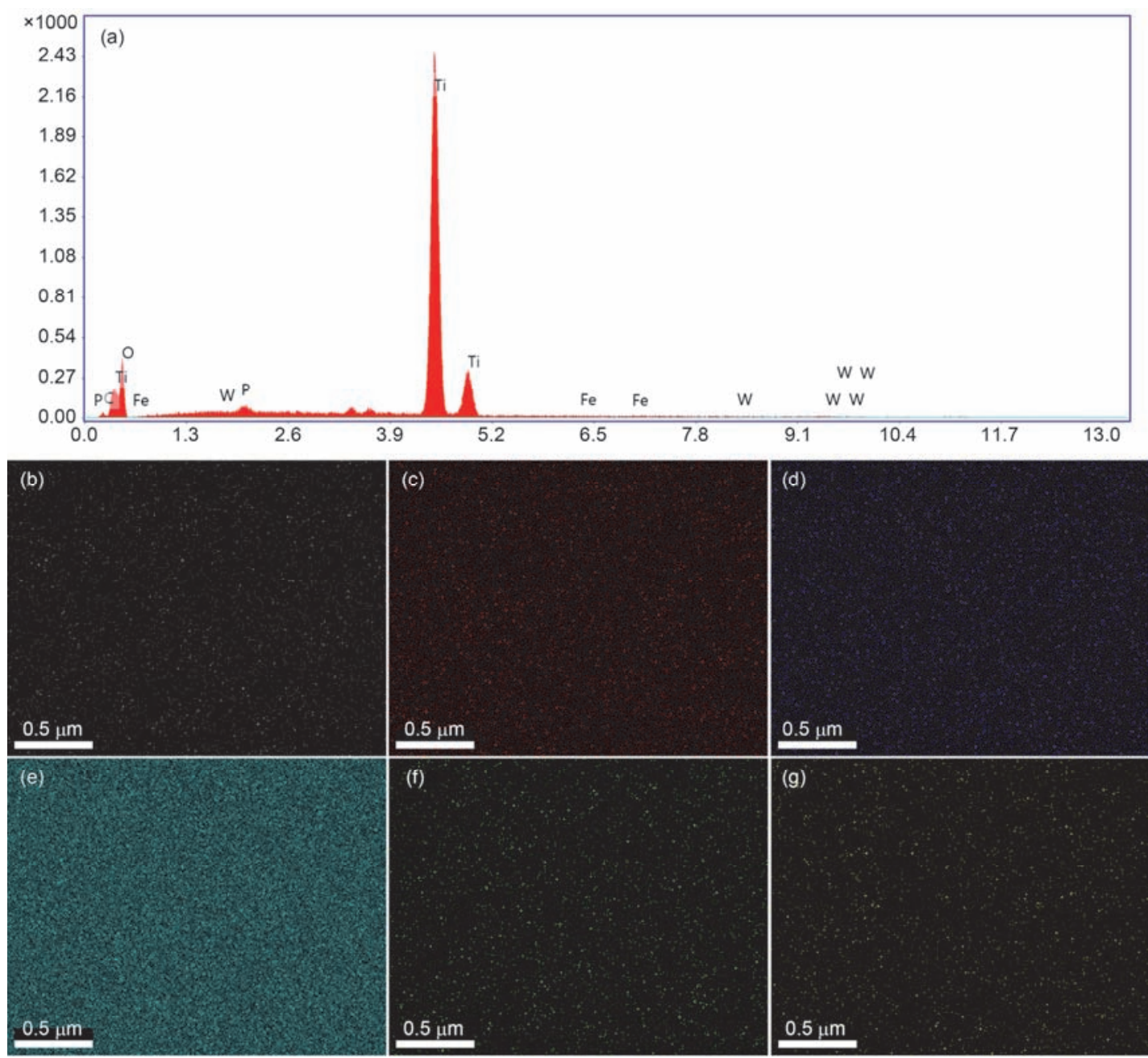

图 3 (网络版彩色)光阳极的形貌表征. (a) $\mathrm{POM} @ \mathrm{TiO}_{2} / \mathrm{P} 25$ 复合材料的EDS图. 元素分布图: (b) C; (c) O; (d) P; (e) Ti; (f) Fe; (g) W

Figure 3 (Color online) Morphological characterization of photoanodes. (a) The EDS spectrum of $\mathrm{POM}_{0} \mathrm{TiO}_{2} / \mathrm{P} 25$ composite material. The element distribution: (b) C; (c) O; (d) P; (e) Ti; (f) Fe; (g) W

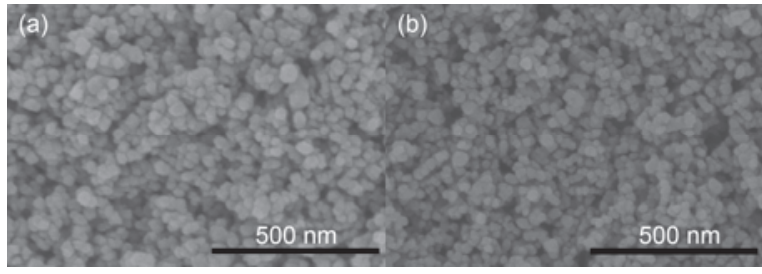

图 4 SEM图. (a) P25 阳极膜; (b) POM@ $\mathrm{TiO}_{2} / \mathrm{P} 25$ 阳极膜

Figure 4 The SEM images. (a) Anode membrane of P25; (b) anode membrane of POM@ $\mathrm{TiO}_{2} / \mathrm{P} 25$

并阻止激发电子重新与染料的空穴复合以及与光阳 极和电解质界面层上的 $\mathrm{I}_{2}$ 反应.

利用IPCE评估了DSSCs对人射光的光电流响应 影响, 如图6(b)所示. 与P25光阳极制作的DSSC相比, $\mathrm{POM} @ \mathrm{TiO}_{2} / \mathrm{P} 25$ 光阳极制作的DSSC具有更宽的响应

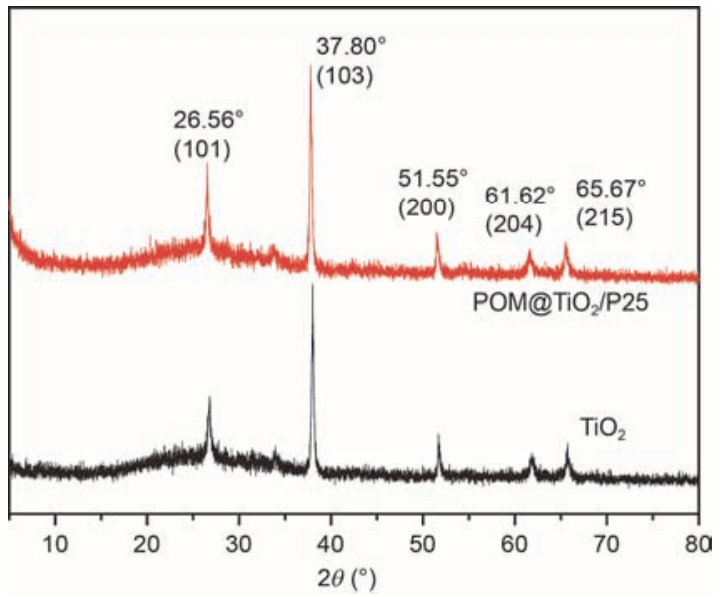

图 5 (网络版彩色)POM@ $\mathrm{TiO}_{2} / \mathrm{P} 25$ 复合材料和锐钛矿 $\mathrm{TiO}_{2}$ 的XRD谱

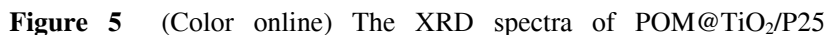
composites and anatase $\mathrm{TiO}_{2}$ 

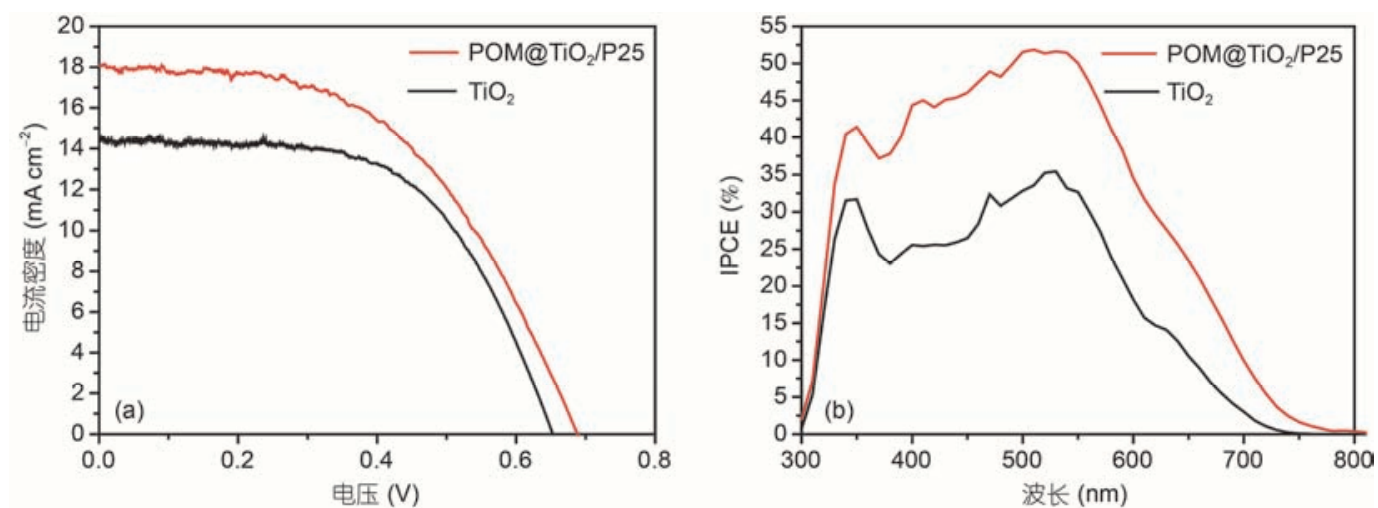

图 6 (网络版彩色)DSSCs的电化学测试. (a) P25 阳极膜与POM@ $\mathrm{TiO}_{2} / \mathrm{P} 25$ 复合材料制作的DSSCs的J-V曲线; (b) P25 阳极膜与POM@ $\mathrm{TiO}_{2} / \mathrm{P} 25$ 复合材料制作的DSSCs的单色光转换效率

Figure 6 (Color online) Electrochemical testing of DSSCs. (a) The $J$ - $V$ curves of DSSCs produced by $\mathrm{P} 25$ anode film and POM@TiO $/ \mathrm{P} 25$ composite material. (b) Single-color optical conversion efficiency of DSSCs made of P25 anode film and POM@TiO $/ \mathrm{P}^{25}$ composite material

\section{表 1 P25 阳极膜与POM@ $\mathrm{TiO}_{2} / \mathrm{P25}$ 复合材料制作的DSSCs的光伏参数}

Table 1 Photovoltaic parameters of DSSCs produced by P25 anode film and POM@ $\mathrm{TiO}_{2} / \mathrm{P} 25$ composite material

\begin{tabular}{lcccc}
\hline & $J_{\mathrm{sc}}\left(\mathrm{mA} \mathrm{cm}^{-2}\right)$ & $V_{\mathrm{oc}}$ & $F F$ & $\eta(\%)$ \\
\hline $\mathrm{P} 25$ & 14.50 & 0.65 & 0.58 & 5.52 \\
$\mathrm{POM} @ \mathrm{TiO}_{2} / \mathrm{P} 25$ 阳极膜 & 18.10 & 0.69 & 0.51 & 6.33 \\
\hline
\end{tabular}
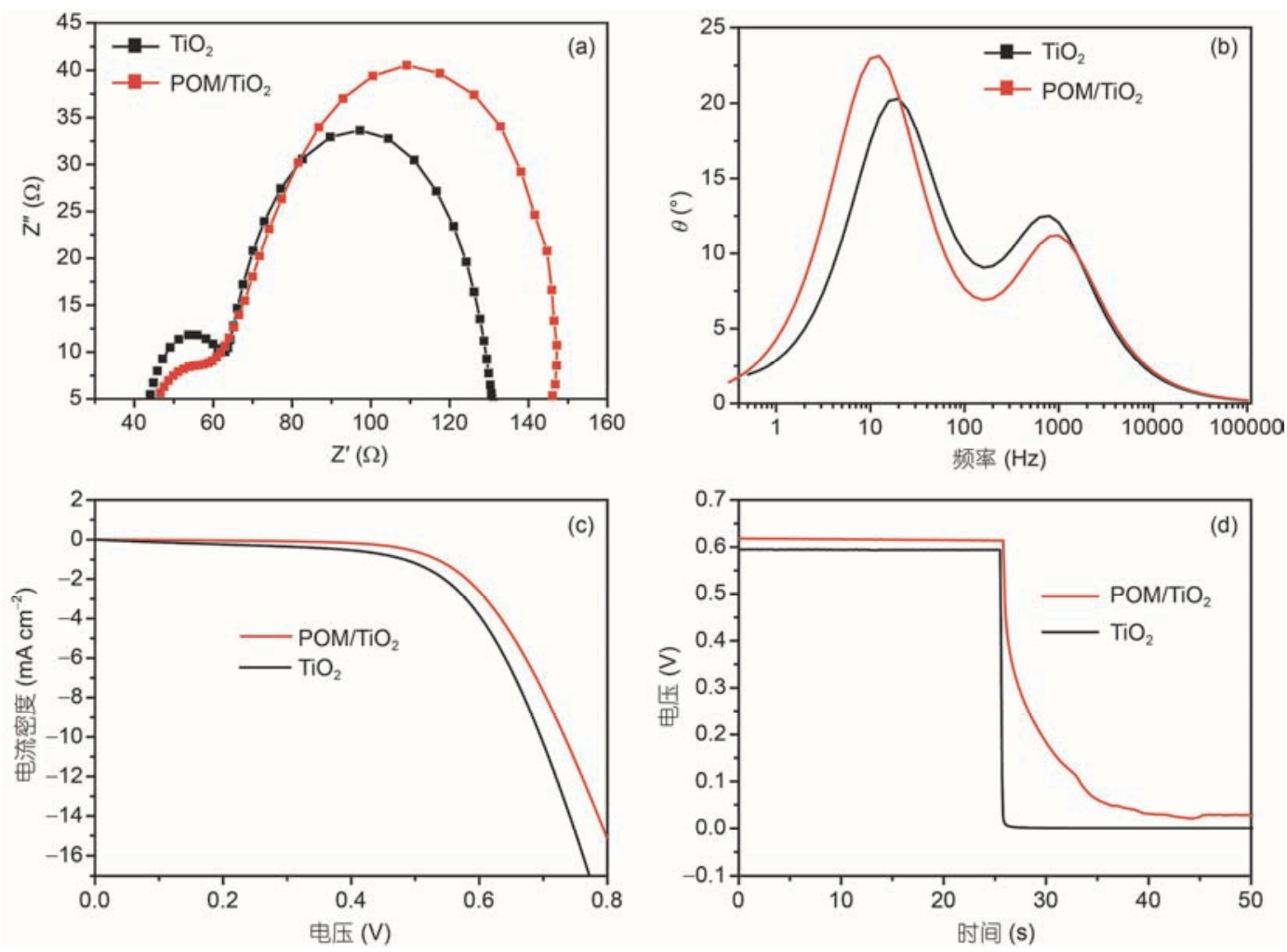

图 7 (网络版彩色)DSSC的电化学阻抗能谱. (a) Nyquist图; (b) Bode图; (c) 黑暗条件下测试的DSSC的光电流密度-电压曲线; (d) DSSCs的电压 衰退曲线

Figure 7 (Color online) Electrochemical impedance spectroscopy of DSSC. (a) Nyquist plot; (b) Bode diagram; (c) the light current density-voltage curve of DSSC tested under dark condition; (d) the voltage decay curve of DSSCs 
范围300 700 nm, 同时在400 800 nm的波长范围内, IPCE值高达到 $53 \%$, 说明多酸的加人增强了光电转 化效率.

EIS测试对于评价电池中相关的电子传递行为的 过程以及研究太阳能电池的内阻十分重要 ${ }^{[24 ~ 26] . ~}$. 对 于DSSCs, EIS的Nyquist图通常呈现的是一个处在高 频区小半圆和一个处在低频区的大半圆. 小半圆表 示电荷转移电阻, 而大半圆反映了电解质界面处的 光生电子传输电阻, 本文主要关注的是经多酸修饰 的 $\mathrm{TiO}_{2}$ 阳极膜中的光生电子转移以及 $\mathrm{POM} @ \mathrm{TiO}_{2} /$ 电 解质界面处的电荷转移即复合电阻 $\left(R_{\mathrm{pt}}\right)$, 所以只涉 及Nyquist图的大半圆. 如图7(a)和(b)所示, 复合膜的 $R_{\mathrm{pt}}$ 更高一些, 说明多酸有效地抑制了电子和空穴的 复合, 使电子可以充分地传递到外电路.

图 7(c) 为黑暗条件下测试的 P25 阳极膜以及 $\mathrm{POM} @ \mathrm{TiO}_{2} / \mathrm{P} 25$ 制作的阳极膜组装成DSSCs的 $J-V$ 特 性曲线. 由图可知, $\mathrm{POM} @ \mathrm{TiO}_{2} / \mathrm{P} 25$ 制作的阳极膜的 电流衰减小于P25阳极膜, 说明夹心型多酸化合物更 有效地抑制了电子-空穴复合.

图 7(d) 为 $\mathrm{P} 25$ 阳极膜以及 $\mathrm{POM} @ \mathrm{TiO}_{2} / \mathrm{P} 25$ 制作的
阳极膜组装成DSSCs 的开路电压衰退曲线. 显然, $\mathrm{POM} @ \mathrm{TiO}_{2} / \mathrm{P} 25$ 制作的阳极膜组装成DSSCs 电压衰 退比P25阳极膜制成的电池要慢, 表明多酸的引入增 长了电子的寿命.

\section{3 结论}

本文根据文献合成夹心型多酸化合物 $\mathrm{K}_{15}\left\{\mathrm{~K}_{3}\right.$ $\left.\left[\left(\mathrm{A}-\alpha-\mathrm{PW}_{9} \mathrm{O}_{34}\right)_{2} \mathrm{Fe}_{2}\left(\mathrm{C}_{2} \mathrm{O}_{4}\right)_{2}\right]\right\} \cdot 29 \mathrm{H}_{2} \mathrm{O}$, 并通过溶胶凝胶 法制备了 $\mathrm{POM} @ \mathrm{TiO}_{2}$ 复合材料. 研究表明, 该多酸化 合物的 LUMO 能级 $(-0.06 \mathrm{~V})$ 低于二氧化钛的导带 $(-0.5 \mathrm{~V})$, 因此多酸化合物可以有效地将染料产生的 光生电子传递到外电路. 此外, 阻抗、暗电流及开路 电压衰退等结果证明了夹心型多酸化合物的加人, 抑制了电子和空穴的复合并增强了电子的寿命, 使 DSSCs的效率得到有效提高 $\left(J_{\mathrm{sc}}=18.10 \mathrm{~mA} / \mathrm{cm}^{2}, V_{\mathrm{oc}}=\right.$ $0.69 \mathrm{~V}, F F=0.51, \eta=6.33 \%)$. 此复合材料获得的 $\mathrm{POM} @ \mathrm{TiO}_{2} / \mathrm{P} 25$ DSSC显示出比P25 DSSC更高的效 率, 说明这项工作不仅为具有分子内电荷转移的多 酸提出了新的应用，同时拓展了染料敏化太阳能电 池的发展空间.

\section{参考文献} 3-14 Science, 2011, 334: 629-634

6 Memarian N, Concina I, Braga A, et al. Hierarchically assembled $\mathrm{ZnO}$ nanocrystallites for high-efficiency dye-sensitized solar cells. Angew Chem Int Ed, 2011, 123: 12529-12533

7 Birkel A, Lee Y G, Koll D, et al. Highly efficient and stable dye-sensitized solar cells based on $\mathrm{SnO}_{2}$ nanocrystals prepared by microwave-assisted synthesis. Energy Environ Sci, 2012, 5: 5392-5400

8 Kim D W, Shin S S, Cho I S, et al. Synthesis and photovoltaic property of fine and uniform $\mathrm{Zn}_{2} \mathrm{SnO}_{4}$ nanoparticles. Nanoscale, 2012, 4: $557-562$

9 Zhang H, Wang Y, Yang D, et al. Directly hydrothermal growth of single crystal $\mathrm{Nb}_{3} \mathrm{O}_{7}(\mathrm{OH})$ nanorod film for high performance dye-sensitized solar cells. Adv Mater, 2012, 24: 1598-1603

10 Zhang H D, Tachibana Y, Kalantarzadeh K. Dye-sensitized solar cells based on $\mathrm{WO}_{3}$. Langmuir, 2010, 24: 19148-19152

11 Burnside S, Moser J E, Grätzel M, et al. Nanocrystalline mesoprous strontium titanate as photoelctrode material for photosensitized solar device: Increasing photovoltage through flatband potential engineering. J Phys Chem B, 1999, 103: 9328-9332

12 Mathew S, Yella A, Gao P, et al. Dye-sensitized solar cells with 13\% efficiency achieved through the molecular engineering of porphyrin sensitizers. Nature, 2014, 10: 1038-1045

13 Hagfeldt A, Grätzel M. Molecular photovoltaics. Acc Chem Res, 2000, 33: 269-277

14 Kikuchi H, Kitano M, Takeuchi M, et al. Extending the photoresponse of $\mathrm{TiO}_{2}$ to the visible light region: Photoelectrochemical behavior of $\mathrm{TiO}_{2}$ thin films prepared by the radio frequency magnetron sputtering deposition method. J Phys Chem B, 2006, 110: 5537-5541 
15 Rhule J T, Hill C L, Judd D, et al. Polyoxometalates in medicine. Chem Rev, 1998, 98: 327-357

16 Parayil S K, Lee Y M, Yoon M. Photoelectrochemical solar cell properties of heteropolytungstic acid-incorporated $\mathrm{TiO}_{2}$ nanodisc thin films. Electrochem Commun, 2009, 11: 1211-1216

$17 \mathrm{Xu} \mathrm{B}, \mathrm{Xu} \mathrm{L}, \mathrm{Gao} \mathrm{G}$, et al. Multicolor electrochromic and $\mathrm{pH}$-sensitive nanocomposite thin film based on polyoxometalates and polyviologen. Electrochim Acta, 2009, 54: 2246-2252

18 Jin G, Wang S M, Chen W L, et al. A photovoltaic system composed of a keplerate-type polyoxometalate and a water-soluble poly ( $p$-phenylenevinylene) derivative. Mater Chem A, 2013, 1: 6727-6731

19 Wang Y J, Chen W L, Chen L, et al. Sandwich-type silicotungstate modified $\mathrm{TiO}_{2}$ microspheres for enhancing light harvesting and reducing electron recombination in dye-sensitized solar cells. Inorg Chem Front, 2017, 4: 559-566

20 Duan Y, Waerenborgh J C, Clemente-Juan J M, et al. Light-induced decarboxylation in a photo-responsive iron-containing complex based on polyoxometalate and oxalato ligands. Chem Sci, 2017, 8: 305-315

21 Li J S, Sang X J, Chen W L, et al. The research of a new polyoxometalates based photosensitizer on dye sensitized solar cell. Inorg Chem Commun, 2013, 38: 78-82

22 Habibi M H, Zendehdel M. Fabrication and characterization of self-assembled multilayer nanostructure titania with high preferential (101) orientation on alumina thin films by layerby-layer dip-coating method. Nanosci Curr, 2010, 6: 642-647

23 Habibi M H, Zendehdel M. Synthesis and characterization of titania nanoparticles on the surface of microporous perlite using sol-gel method: Influence of titania precursor on characteristics. J Inorg Organomet Polym, 2011, 21: 634-639

24 Li J S, Sang X J, Chen W L, et al. Enhanced visible photovoltaic response of $\mathrm{TiO}_{2}$ thin film with an all-inorganic donor-acceptor type polyoxometalate. ACS Appl Mater Interfaces, 2015, 7: 13714-13721

25 Fabregat S F, Garcia B G, Mora S I, et al. Characterization of nanostructured hybrid and organic solar cells by impedance spectroscopy. Chem Chem Phys, 2011, 13: 9083-9118

26 Goes M S, Joanni E, Muniz E C, et al. Impedance spectroscopy analysis of the effect of $\mathrm{TiO}_{2}$ blocking layers on the efficiency of dye-sensitized solar cells. J Phys Chem C, 2012, 116: 12415-12421 


\title{
Application of sandwich-type polyoxometalates modified $\mathrm{TiO}_{2}$ in dye-sensitized solar cells
}

\author{
He Zhang, Jian-Ping Li, Wei-Lin Chen * \& En-Bo Wang* \\ Key Laboratory of Polyoxometalates Science Ministry of Education in Northeast Normal University, ChangChun 130024, China \\ *Corresponding authors, E-mail: chenwl@nenu.edu.cn; wangeb889@nenu.edu.cn
}

Nowadays, the rapid consumption of fossil energy has led to global energy shortage. At present, more than $80 \%$ of the energy consumed globally is derived from non-renewable fossil fuels such as coal, oil and natural gas. The burning of fossil fuels will also bring about a series of problems such as the greenhouse effect, the falling quality of global air, the formation of acid rain and the pollution of water bodies. Therefore, whether new energy sources that can replace fossil energy can be found has become an increasingly popular concern problem. Dye-sensitized solar cells (DSSCs) as a new type of renewable energy source have attracted wide attention in recent years, because of their simple fabrication process, low production costs, relatively high photoelectric conversion efficiency (PCE), and being environmentally safe. But the recombinations of photogenerated electrons holes are seriously affected the photoelectric conversion efficiency of DSSCs. Polyoxometalates (POMs) are a class of metal oxide clusters with the highest oxidation state of tungsten, molybdenum or vanadium. Due to their structural diversity and unique physicochemical properties, they have been applied in catalysis, photochemistry, biology and medicine. However, due to POMs have excellent photoelectrochemical properties, which can be used as an excellent electronic extractant in the photoanode of DSSCs. Here, the sandwich-type polyoxometalates compound $\mathrm{K}_{15}\left\{\mathrm{~K}_{3}\left[\left(\mathrm{~A}-\alpha-\mathrm{PW}_{9} \mathrm{O}_{34}\right)_{2} \mathrm{Fe}_{2}\left(\mathrm{C}_{2} \mathrm{O}_{4}\right)_{2}\right]\right\} \cdot 29 \mathrm{H}_{2} \mathrm{O}$ with the intramolecular electron transfer properties is composited with $\mathrm{TiO}_{2}$ by sol-gel method to prepare a $\mathrm{POM} @ \mathrm{TiO}_{2}$ composite photoanode. The energy band structure of the polyoxometalate is studied by optical and electrochemical measurements. The result shows that the LUMO energy level $(-0.06 \mathrm{~V})$ of the sandwich-type polyoxometalates is lower than that $\mathrm{TiO}_{2}$ and the optical band gap is $2.28 \mathrm{eV}$.

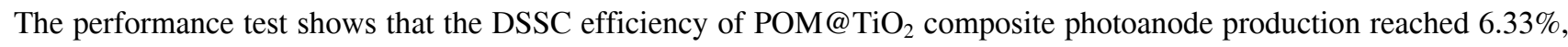
which is $15 \%$ higher than that of battery whose anode is pure $\mathrm{TiO}_{2}(5.52 \%)$. Electrochemical impedance spectroscopy (EIS), dark current tests and voltage decay curve tests demonstrate that sandwich-type POMs effectively suppress electron-hole recombinations and increase electron lifetime. The incident monochromatic photon-electron conversion efficiency (IPCE) test further demonstrates that the introduction of the POMs increases the monochromatic conversion efficiency (from $35 \%$ to $53 \%$ ).

dye-sensitized solar cells, polyoxometalates, anode, electrons transmitting, the recombinations of photogenerated electrons and holes

doi: 10.1360/N972018-00632 